Journal of Bangladesh Academy of Sciences, Vol. 37, No. 1, 57-63, 2013

\title{
PREVALENCE AND ANTIBIOGRAM PROFILE OF UROPATHOGENS ISOLATED FROM HOSPITAL AND COMMUNITY PATIENTS WITH URINARY TRACT INFECTIONS IN DHAKA CITY
}

\author{
AFSANA FATEMA NOOR ${ }^{1}$, FARIZA SHAMS ${ }^{1}$, SAURAB KISHORE MUNSHI ${ }^{1}$, \\ MUNIR HASSAN AND RASHED NOOR ${ }^{* 1}$
}

Ibn Sina Diagnostic and Imaging Center, Dhanmondi 9/A, Dhaka-1209, Bangladesh

\begin{abstract}
Urinary tract infection (UTI) is increasingly rising in Bangladesh due to the development of resistance of causative pathogens against commonly prescribed antibiotics. Present study attempted to examine the prevalence of such uropathogens and their antibiogram profiles. Among 462 urine samples collected from patients with UTI, 100 were found to be culture positive. Escherichia coli (70\%) was predominating, while Klebsiella spp. (11\%) and Enterococcus spp. (6\%) were also prevalent. About $96 \%$ uropathogens were sensitive against imipenem, and $75 \%$ against amikacin. The resistance rate of E. coli and Klebsiella spp. against ampicillin was 98.5 and $100 \%$, respectively, and to cefotaxime, 84.3 and $72.8 \%$, respectively. More than $80 \%$ resistance against these antibiotics was scored for other isolates. The frequency of drug resistance was found to be comparatively elevated in E. coli, Pseudomonas spp. and Proteus spp. Overall, the present investigation emphasized the need for routine screening of antibiotic resistance to promote effective medication against UTI.
\end{abstract}

Key words: Urinary tract infection, Pathogenic microorganisms, Antibiotic resistance

\section{INTRODUCTION}

Urinary tract infection (UTI) involves the infection of kidneys, ureters, bladder, or urethra by pathogenic invasion of the urinary tract, which ultimately leads to an inflammatory response of the urothelium. All individuals may be susceptible to UTIs; however, the prevalence of infection differs with age, sex and certain predisposing factors (Griebling 2001). The incidence of infection is greater in females than in males with two exceptions, infants and the catheter related infections (Kunin 1987, Pezzlo 1988). Women tend to get UTIs more often because their urethra is shorter and closer to the anus than men and hence the pathogenic bacteria get quick access to the bladder.

UTI is considered significant and requires treatment when more than 105 microorganisms per $\mathrm{ml}$ of urine are present in a properly collected sample (Travis and Burhard 1996, Talkoff and Rubin 1986). Uropathogenic Escherichia coli causes $90 \%$ of

*Corresponding author: <noor.rashed @yahoo.com>.

${ }^{1}$ Department of Microbiology, Stamford University Bangladesh, Dhaka-1217, Bangladesh. 
the UTIs in anatomically-normal, unobstructed urinary tracts (Behzadi and Behzadi 2006, Todar 2008). After Escherichia coli, the most common UTIs pathogens include $S$. saprophyticus, Enterococcus spp., Pseudomonas aeruginosa, Candida spp., Klebsiella pneumonia, Proteus spp. and Enterobacter spp. Group B streptococci are uncommon pathogens in UTIs in young healthy women (Singleton and Sainsbury 2001). Interestingly, the pathogens traditionally associated with UTI are known to change many of their features, particularly due to their antimicrobial resistance patterns (Ranold 2003). Though antibiotics are the mainstay treatment for all UTIs, the increasing trend of resistance in bacterial pathogens is of worldwide concern that can vary according to geographical and regional locations (Mathai et al. 2001).

Since the initiation of antimicrobial therapy in UTI is empirical, a huge need demand for antimicrobial resistance exists at local, national and international levels (Bassetti 2000). Knowledge on the antimicrobial resistance patterns of common uropathogens and the subsequent treatment are thus required to minimize urinary diseases (Prais et al. 2003). Along these lines, the present study was designed to identify the etiological agents of UTI in males and females of different age groups and to investigate their responses against locally available antibiotics commonly prescribed by the physicians.

\section{MATERIALS AND METHODS}

Urine specimens were collected from 462 patients of all ages suffering from UTIs for this study between January 2009 and July 2009 and the samples were analyzed at Microbiology laboratory of Ibn Sina Diagnostic and Imaging Center. After collecting the specimens aseptically, samples were centrifuged and examined microscopically to detect the presence of white cells (puss cells), red cells, yeast cells, epithelial cells, parasites, casts, crystals etc. For bacteriological analysis, MacConkey agar (MAC) and Blood agar media were used. With a calibrated loop of $3 \mathrm{~mm}$ diameter, the appropriately labeled media plates were streaked aseptically with $0.01 \mathrm{ml}$ urine sample. The plates were then incubated aerobically at $37^{\circ} \mathrm{C}$ for $24 \mathrm{hrs}$. Bacterial identification was done by examination of the overnight culture and Gram-staining. Standard biochemical tests were performed also to identify the bacteria of interest (Cappuccino and Sherman 1996). Agar disc diffusion assay was used to determine the bacterial susceptibility to different antibiotics towards bacteria in vitro as described previously (Bauer et al. 1966). The zone diameters for individual antimicrobial agents were translated into susceptible, intermediate and resistant categories (Ferraro 2001).

\section{RESULTS AND DISCUSSION}

Prevalence of UTI according to age groups and gender: Out of 462 urine specimens from UTI affected children and adults, $79 \%$ were female and $21 \%$ were male. Women in 
the age group 16 - 45 years are most likely to suffer from UTI (Fig. 1). Among the elderly, after 45 years males became more prone to UTI. The number of male patients increased significantly in the age group 46 years and above than the age group $16-45$ years. Prostatic gland enlargement and decrease of bacteriostatic prostatic secretions might account for such infections.

Table 1. Frequency of microorganisms isolated from 100 positive urine positive cultures $(\mathbf{n}=\mathbf{1 0 0})$.

\begin{tabular}{lcc}
\hline Microorganisms & No. of isolates & \% frequency \\
\hline Gram-negative rod & 70 & \\
Escherichia coli & 11 & 70.0 \\
Klebsiella spp. & 4 & 11.0 \\
Pseudomonas spp. & 2 & 4.0 \\
Proteus spp. & & 2.0 \\
Gram-positive coccus & 6 & 6.0 \\
Enterococcus spp. & 3 & 3.0 \\
Staphylococcus saprophyticus & 2 & 2.0 \\
Staphylococcus aureus & & \\
Fungus & 2 & 2.0 \\
Candida spp. & 2 & \\
\hline
\end{tabular}

Antibiogram profile of the isolates causing UTI: The drug resistance and sensitivity profile of the organism showed that all the bacterial isolates were resistant against commonly used antibiotics, e.g. ampicillin, cefotaxime and cefixime (Fig. 2).

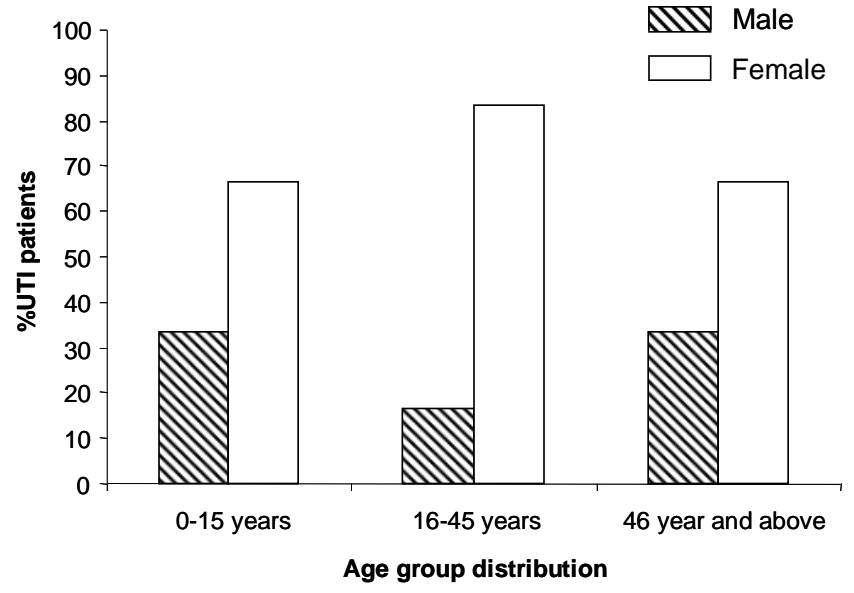

Fig. 1. Age group and gender distributions of the patients suffering from UTI. Female patients (79\%) were predominant as shown by the white bars over the male patients $(21 \%)$ mostly in the age group of $16-45$ years as shown by the stripped bars.

Moreover, more than $60 \%$ of the organisms showed resistance against cotrimoxazole, nalidixic acid, nitrofurantoin, ciprofloxacin and ceftriaxone. Increasing resistance rate to all antibiotics used in this study with the exception of imipenem and amikacin may 
be explained as uncontrolled consumption of these antibiotics during the past decade in our region (Hiller et al. 2002). The inordinate accessibility of antibiotics in shops and open markets is probably an important factor in antibiotic resistance worthy of consideration. Sometimes discontinuation of treatment due to negligence of patient or unavailability of drugs develops antibiotic resistance. The results of this study, therefore support the use of either imipenem or amikacin as a reasonable choice for empiric therapy in uncomplicated UTI, particularly in the community setting.

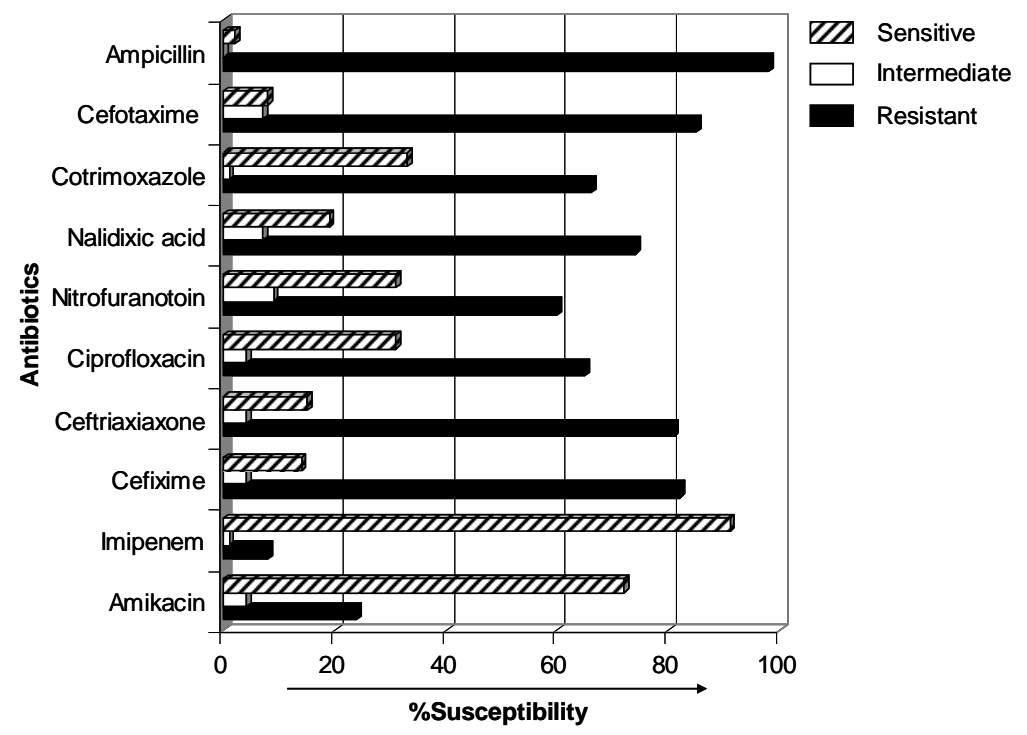

Fig. 2. Antimicrobial resistance and sensitivity profile of the 98 clinical bacterial isolates recovered from patients suffering from UTIs. The highest resistance was observed against ampicillin (98.5\%) as shown by black bar. Most of the isolates $(96 \%)$ were found to be sensitive against imipenem as shown by stripped bar.

The majority of the pathogenic microorganisms isolated in this study belonged to the Enterobacteriaceae family. As shown in Table 1, E. coli was identified as the most common causative agent of UTIs (70\% of total isolates in both sexes), which is in consistence with the findings of Bhowmick and Rashid (2004). Since E. coli caused the majority of UTI, the emergence of antibiotic resistant $E$. coli thus presents a challenge for urinary tract health management. A recent study on college women at Duke University showed that $29.6 \%$ of the E. coli samples isolated from urine cultures were resistant to trimethoprim-sulfamethoxazole, which is a standard antibiotic combination used for UTI. A study in Taiwan found that prior exposure to ciprofloxacin raises the risk of resistance by a factor of 13 (Song 2009). In this study, the range of resistance of E. coli to ampicillin was $98.5 \%$ and to cefotaxime, nalidixic acid and cefixime were $84.3,82.9$ and $80 \%$, respectively (Fig. 3). These resistance rates were very high. Therefore, these drugs should 
no longer be prescribed as initial empirical therapy in our region. E. coli also showed significant resistance against cotrimoxazole and ciprofloxacin (60 - 75\%). The susceptibility pattern of $E$. coli against imipenem and amikacin was very significant (92.8, and $78.5 \%$, respectively).

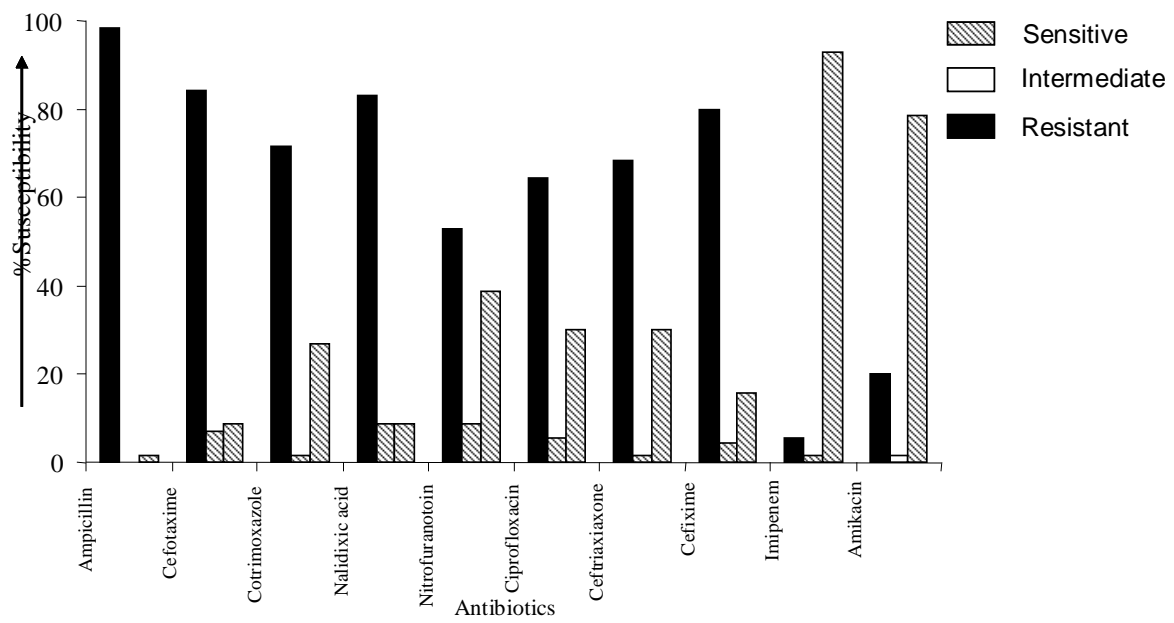

Fig. 3. Antibiotic susceptibility pattern of $E$. coli isolates. The isolates were highly resistant against most of the tested antibiotics as other pathogenic isolates. Imepenem and amikacin found to be more effective against the isolates (as shown by stripped bar).

Klebsiella spp. was the second most prevalent organisms isolated from UTI cases. The isolates were highly resistant against ampicillin (100\%) and against cefotaxime, nalidixic acid, nitrofurantoin, and cefixime ranging between 60 and 70\%. However, this pathogen showed 100\% sensitivity against imipenem and $91 \%$ sensitivity against amikacin. Besides, Proteus spp. isolates were 100\% resistant against most of the antibiotics. All the Pseudomonas isolates were found $100 \%$ resistant against ampicillin, cefotaxime, cotrimoxazole, nalidixic acid, nitrofurantoin and cefixime. Higher resistance also found against ciprofloxacin, ceftriaxone, imipenem and amikacin.

Among the Gram-positive coccus, isolates of Enterococcus spp. showed 100\% resistance against cotrimoxazole, cefixime and amikacin. The resistance rate against ampicillin, ciprofloxacin and cefotaxime (83.4\%) were also high. Among antibiotics used in this investigation, Enterococcus spp. isolates were very sensitive against imipenem $(100 \%)$ and nalidixic acid $(83.4 \%)$.

Staphylococcus saprophyticus was found highly resistant against ampicillin, cefotaxime and cefixime. The rate of sensitivity to nalidixic acid, imipenem and amikacin among these isolates were $100 \%$ and above $60 \%$ against ciprofloxacin. All the S. aureus isolates showed high sensitivity (100\%) against ampicillin, cefotaxime, ciprofloxacin and 
cefixime. The resistance rate against nalidixic acid, nitrofurantoin and ceftriaxone were moderate (50\%).

Although in least amounts, but some Candida spp. were found to be present in the urinary samples. The challenge comes from the fact that Candida spp. in the urine can be a marker of invasive renal parenchymal disease related to disseminated candidiasis or postlaparotomy peritonitis (Bensousan 1994).

\section{CONCLUSION}

Antibiotic resistance is becoming a big problem for the public health which threatens the lives of hospitalized individual as well as those with chronic conditions and adds considerably to health care cost. The relatively high resistance of isolates to commonly used antibiotics as observed in this study is worrisome, especially in the developing countries like Bangladesh where most of these antibiotics still serve as first line drugs. Therefore, routine sensitivity screening of antibiotics before prescription is suggested.

\section{ACKNOWLEDGEMENTS}

This work was financed by Stamford University Bangladesh and Ibn Sina Diagnostic and Imaging Center, Dhaka.

\section{REFERENCES}

Bassetti, D., M. Bassetyti and M. Mantero. 2000. Strategies for antibiotic selection in empirical therapy. Clin. Microbiol. Infect. 6: 98-100.

Bauer, A.W., W. M. M. Kirby, J. C. Sherris and M. Tierch. 1966 Antibiotic susceptibility testing by a standardized single disc method. Am. J. Clin. Pathol. 45(4): 493-496.

Behzadi, P. and E. Behzadi. 2006. Microbiology of Prokaryotes. 1st ed. Islamic Azad University Press. pp. 101.

Bhowmick, B. K. and H. Rashid. 2004. Prevalence and antibiotic susceptibility of E. coli isolated from urinary tract infection (UTI) in Bangladesh. Pakistan J. Biol. Sciences 7(5): 717-720.

Cappuccino, J. G. and N. Sherman. 1996. Microbiology- A Laboratory Manual. 4th ed. The Benjamin/Cummings Publishing Co., Inc., Menlo Park, California.

Ferraro, M. J., W. A. Craig and M. N. Dudley. 2001. Performance standards for antimicrobial susceptibility testing - NCCLS informational supplement. 11th ed. NCCLS, Pennsylvania, USA.

Griebling, T. L. 2001. Urinary Tract Infection in Women. In: Urologic Diseases in America. (Ed. Litwin, M. S. and C. S. Saigal). US Government Printing Office, Washington, DC. pp. 587.

Hillier, S. L., J. T. Magee, A. J. Howard and S. R. Palmer. 2002. How strong is the evidence that antibiotic use is risk factor for antibiotic-resistant, community acquired urinary tract infection? J. Antimicrob. Chemother. 50: 241-247.

Kunin, C. M. 1987. Detection, Prevention and Management of Urinary Tract Infections. 4th ed. Lea \& Febiger, Philadelphia. 
Mathai, D., R. N. Jones and M. A. Pfaller. 2001. Epidemiology and frequency of resistance among pathogens causing urinary tract infection in 1,510 hospitalized patients : A report from the SENTRY antimicrobial surveillance program (North America). Diagn. Microbiol. Infect. Dis. 40: $129-136$.

Pezzlo, M. 1988. Detection of Urinary Tract Infections by Rapid Methods. Clinical Microbiol. Rev 1(2): 268-280.

Prais, D., R. Straussberg, Y. Avitzur, M. Nussinovitch, L. Harel and J. Amir. 2003. Bacterial susceptibility to oral antibiotics in community acquired urinary tract infection. Arch. Dis. child. 88(3): 215-218.

Ronald, A. 2003. The etiology of urinary tract infection : Traditional and emerging pathogens. Dis Mon. 49(2): 71-82.

Singleton, P. and D. Sainsbury. 2001. Dictionary of Microbiology and Molecular Biology. 3rd ed. John Wiley \& Sons, New York. pp. 812.

Song, S. 2009. Antibiotic resistance mechanisms of Escherichia coli isolates from urinary specimens. Korean J. Lab. Med. 29(1): 17-24.

Todar, K. 2008. Pathogenic Escherichia coli. In: Todar's Online Text Book of Bacteriology. University of Wisconsin-Madison.

Tolkoff, N. E. and R. H. Rubin. 1986. Urinary tract infection : Significance and management. Bull. NY. Acad. Med. 62(2): 131-148.

Travis, L. B. and B. H. Bruhard. 1996. Infections of the urinary tract. In: Rudolph's Pediatrics (Ed. Rudolph, A. M.). 20th ed. Appleton \& Lange, Stanford, Connecticut. pp. 1388-1392.

(Received revised manuscript on 25 March, 2013) 